Das Verdienst der Arbeit besteht vor allem darin, dass sie den Kenntnisstand der Wahlforschung in einem für das Wahlverhalten in der Bundesrepublik wesentlichen Teil erheblich erweitert. Einer breiteren Leserschaft dürfte das Buch in seiner sprachlich eingängigen Art zudem die große Zahl der möglichen Faktoren nahe bringen, die zum Abschmelzen ländlich-katholischer Hochburgen beigetragen haben. Die politikwissenschaftliche Wahlforschung wird sich gleichwohl mit den Schwächen der Ursachenanalyse nicht zufrieden geben können. Wackers' Arbeit ist somit ohne Zweifel ein wichtiger Anstoß, sich der generellen Frage nach dem Verhältnis von älteren, meist regional verankerten sozialstrukturellen beziehungsweise religiös-kulturellen Bindungen einerseits und den zunehmend volatilen Wahlentscheidungen der Individuen andererseits zu stellen. Den langen Schatten des katholischen Milieus und die anhaltende Bedeutung konfessioneller und religiöser Unterschiede für das gegenwärtige Wahlverhalten in Deutschland kann und wird man dabei nicht übersehen.

Antonius Liedhegener

\title{
Ländervergleichende Studie zu Auswirkungen des Nichtwählens auf Demokratien
}

\author{
de Nève, Dorothée: NichtwählerInnen - eine Gefahr für die Demokratie?, Verlag Barbara \\ Budrich, Opladen / Farmington Hills 2008, 238 Seiten, € 24,90.
}

Wahlen sind ein zentraler Bestandteil politischer Repräsentation. Wer wählen geht, ist politisch aktiv. Doch gilt auch der Umkehrschluss? Und welche potentiellen Auswirkungen hat das Nichtwählen auf die „Qualität der Demokratie“ (S. 15)? Dorothée de Nève beschäftigt sich aus Perspektive der Nichtwählerforschung sekundäranalytisch mit einer Thematik, die für einen über das politikwissenschaftliche Fachpublikum hinausgehenden Leserkreis von Interesse sein dürfte, denn die Wahlbeteiligung scheint in den vergangenen Jahrzehnten in den meisten westlichen Demokratien stetig und irreversibel zu sinken. Welchen Standpunkt die Autorin gegenüber ihrem Forschungsthema einnimmt, wird sichtbar, wenn sie zu Beginn der Ausführungen die Entscheidung, nicht wählen zu gehen, als „Resignation an der Wahlurne" (S. 18) bewertet. Aus Sicht der "Theorie der liberalen Demokratie“ (S. 51), auf die sich de Nève beruft, kann die Nichtbeteiligung an Wahlen jedoch durchaus auch als positiv, zumindest aber als funktionell vereinbar mit einer demokratischen Herrschaftsstruktur interpretiert werden.

Vor die Diskussion verschiedener Nichtwählertypologisierungen (S. 31 - 37) stellt de Nève die Unterscheidung zwischen systematischer Exklusion aufgrund wahlrechtlicher Regelungen, struktureller Exklusion im Zusammenhang mit „informelle(n) Exklusions- beziehungsweise Diskriminierungsmechanismen“, individuell motiviertem Wahlverzicht und unechter Nichtwahlteilnahme durch „technische Pannen der Wahladministration“ (S. 29 - 31). Leider folgt die Autorin dieser theoretisch zwar einleuchtenden, in der Praxis aber wohl nur schwerlich überprüfbaren Typologisierung zwischen einer strukturellen Exklusion bei Wahlen, zum Beispiel von ethnischen Minderheiten, und eines individuellen Wahlverzichts in ihren weiteren Ausführungen nicht systematisch und stringent genug (zum Beispiel S. 71, 81, 140, 201 f.), obwohl gerade dieser Aspekt für die Nichtwählerforschung von zentralem Interesse sein dürfte. Es folgt ein longitudinaler Vergleich von Wahlbeteili- 
gungen für die Zeit nach dem Ende des Zweiten Weltkriegs bis zum Jahr 2007 (S. 37 - 44). Untersuchungseinheiten sind nationale Wahlen in Belgien, Italien, den Niederlanden, Dänemark, Schweden, Deutschland, Norwegen, Finnland, Tschechien, Frankreich, Portugal, Großbritannien, Spanien, Bulgarien, Ungarn und der Schweiz. Welche Überlegungen der Fallauswahl zu Grunde liegen, wird allerdings nicht erörtert. Somit bleibt auch offen, warum die Thematik nicht ebenfalls am Beispiel der USA als der weltweit ersten und ältesten repräsentativen Demokratie in einem Flächenland mit bekanntermaßen geringen Wahlbeteiligungsquoten eingehend untersucht wird. Nicht analysiert werden ferner Bestimmungsfaktoren, Folgen und Wechselwirkungen unterschiedlicher Partizipationswerte bei Wahlen auf den verschiedenen Ebenen politischer Systeme.

Die anfangs von de Nève skizzierte Problemstellung wird während der weiteren Ausführungen deutlicher herausgearbeitet. Aus der Frage, „inwiefern [...] (die Nichtwahl) die Funktionslogik demokratischer Systeme tatsächlich stört" (S. 18, Hervorhebung durch den Rezensenten) wird eine nach der hypothetischen „Gefahr für die Demokratie“ (S. 52). Das bedeutet, dass nicht die tatsächlichen Auswirkungen der Wahlabstinenz untersucht werden sollen, sondern die potentiellen. Gefahr drohe erstens, wenn „die systematische Exklusion [...] und/oder die Wahlenthaltung der Wahlberechtigten ein solches Ausmaß erreichen, dass das Mehrheitsprinzip in Frage gestellt wird“ (S. 52), zweitens durch eine signifikant sozialstrukturelle Abweichung der Nichtwähler von den Wählern, da dann das Gleichheitsprinzip verletzt würde und drittens bei Vorliegen „demokratiefeindliche(r) Einstellungen“" (S. 52) der Nichtwähler.

Empirisch wird zunächst die systematische Exklusion dargestellt (S. 60 - 66). Es folgt für ausgewählte Staaten die Darstellung der Nichtwähleranteile (S. 66 - 71), wobei dies mit absoluten Zahlen geschieht. Mögliche Entwicklungen in der Bevölkerungsgröße eines Staates können so nicht einbezogen werden. Im Hauptteil der Arbeit (viertes und fünftes Kapitel) werden Ausprägungen gängiger sozialstruktureller Variablen, politischer Einstellungen und politischer Partizipationswerte von Wählern und Nichtwählern paarweise miteinander verglichen. Datengrundlage sind 39 Nachwahlstudien (Erhebungen zwischen 1990 und 2001), die nach Staaten kumuliert werden (S. 83 f.). Methodisch werden die zugrunde gelegten Hypothesen mit Instrumenten der deskriptiven Statistik untersucht. Die Autorin weist auf Einschränkungen der Aussagekraft des verfügbaren Datenmaterials hin, die zum Teil zurückzuführen sind auf niedrige Befragtenzahlen und vor allem auf das so genannte "overreporting" (S. 28, 83), das heißt, nicht jeder Wahlberechtigte räumt bei einer Nachwahlbefragung ein, nicht gewählt zu haben.

Die Ergebnisse der Untersuchung können aufgrund einer hohen Varianz zwischen den untersuchten Staaten kaum zu einem bestimmten Muster zusammengefasst beziehungsweise generalisiert werden: In Bezug auf die untersuchten soziostrukturellen Merkmale sind „die Befunde heterogen“ und die „sozialstrukturelle Exklusion [...] länderspezifisch unterschiedlich" (S. 138). Zu den politischen Einstellungen kann de Nève aber durchaus zeigen, dass Nichtwähler gegenüber Wählern durch ein stärkeres politisches Desinteresse gekennzeichnet sind und sie „ihre Wahlenthaltung nicht beziehungsweise nur in sehr eingeschränktem Maße durch andere Formen der politischen Partizipation kompensieren" (S. 170).

Insgesamt gibt das Buch trotz einiger Kritikpunkte einen guten Überblick über die Entwicklung der Anteile der Wahlberechtigten an der Gesamtbevölkerung (tendenzielle Zunahme) und der Wahlbeteiligung (tendenzielle Abnahme) in den untersuchten Staaten. Je nach Datenverfügbarkeit werden interessante Details zu Unterschieden und Gemeinsamkei- 
ten zwischen Nichtwählern und Wählern hinsichtlich soziostruktureller Eigenschaften (S. 86 - 141) und politischer Einstellungen (S. 143 - 199) herausgearbeitet. Der Erkenntnisgewinn zur Frage, ob Nichtwähler eine Gefahr für die Demokratie darstellen, fällt demgegenüber weniger ergiebig aus. Der um Ausgewogenheit bemühten Würdigung des Nichtwählens als „Krisensymptom“ (S. 204) oder als Gefährdung der „demokratische(n) Qualität“, jedoch nicht als Gefahr für die „,institutionelle Ordnung“ (S. 206), kommt einige Plausibilität zu. Sie bleibt aber gleichzeitig vage und sowohl empirisch als auch argumentativ nicht durchgängig nachvollziehbar. Mit der Empfehlung, „bewährte und neue gesellschaftliche und politische Inklusionsstrategien zu nutzen, um die Kongruenz zwischen Beteiligten und Betroffenen (wieder-)herzustellen“ (S. 207), schließt de Nève ihre Studie ab. Wie diese Strategien, vor dem Hintergrund eines gestiegenen Niveaus an politischer Unübersichtlichkeit in politischen Mehrebenensystemen (institutionelle und inhaltliche Interdependenzen, Verhandlungscharakter politischer Entscheidungsprozesse, damit einhergehend Intransparenz und unklarere politische Verantwortungszuschreibung) aussehen und implementiert werden können, wird eine wichtige Aufgabe weiterer Forschungsarbeiten sein.

Benjamin Höhne

\section{Neugefasstes Standardwerk zur Parteienfinanzierung}

\section{Sickinger, Hubert: Politikfinanzierung in Österreich. Ein Handbuch, Czernin Verlag, Wien} 2009, 527 Seiten, € 39,-.

Für die komparatistische Analyse eines international selten bearbeiteten Themenfeldes (und die Finanzen politischer Parteien sind ein in der Parteienforschung durchweg vernachlässigter Aspekt) haben sich vier aufeinander folgende Schritte als nützlich erwiesen. Zunächst bereiten mehr oder weniger kompatible Aufsatzsammlungen das Feld vor, dann folgen ausgefeilte Studien einzelner Länder, später vergleichende Arbeiten über Ländergruppen. Schließlich wird eine Zusammenfassung und Systematisierung der so gewonnenen Einsichten möglich. Als das hier angezeigte Werk erstmals erschien, war sein Verfasser bereits durch das Buch „Politisches Geld“ (1990, mit Rainer Nick) als profunder Kenner der Materie ausgewiesen. Das neue Buch von Hubert Sickinger war und ist nicht nur umfangreich, sondern auch umfassend. Das gilt ganz besonders für die breite, im besten Sinne erschöpfende, aber nicht ermüdende Abhandlung aller Teilaspekte des Themas. Als Oberbegriff für die monetär quantifizierbaren Kosten der Parteiendemokratie umfasst Politikfinanzierung neben den (facettenreich dargebotenen) Parteifinanzen auch Politikergehälter und die Kosten der parlamentarischen Infrastruktur.

Mit seinem Handbuch stellte sich Sickinger schon 1997 in eine Reihe mit den Klassikern des Genre: Alexander Heard (USA, 1960), Khayyam Z. Paltiel (Kanada, 1970), Uwe Schleth (Deutschland, 1973) und Michael Pinto-Duschinsky (Großbritannien, 1981) hatten die Stufe der länderspezifischen Intensivstudien bereits überzeugend eingeleitet. Später ergänzten dann Stefan Hofmann (Spanien, 1998), Marcin Walecki (Polen, 2005) und Martin Schurig (Frankreich, 2006) die Reihe der für einen fundierten Vergleich von Parteifinanzen unverzichtbaren Länderstudien. Jetzt legt Sickinger eine grundlegend überarbeitete und vollständig aktualisierte Neufassung seines Standardwerks über Österreich vor. 